\title{
Patrones de crecimiento postnatal en ocho especies de garzas (Ciconiiformes: Ardeidae)
}

\author{
Dennis Denis Ávila \\ Facultad de Biología, Universidad de La Habana. Calle 25 entre J e I, Vedado, Ciudad de La Habana, CP 3200, Cuba; \\ dda@fbio.uh.cu
}

\author{
Recibido 09-VII-2010. Corregido 03-XI-2010. Aceptado 01-XII-2010.
}

\begin{abstract}
Postnatal growth patterns in eight species of herons and egrets (Ciconiiformes: Ardeidae). Avian postnatal growth has received considerable attention and its ecological implications have been deeply analyzed. In this current paper, I describe the patterns of culmen and tarsus growth, as well as of weight gain patterns in eight species of herons and egrets (Aves: Ardeidae) found in the Birama Swamp in Eastern Cuba. Between 1998 and 2006, 714 nestlings of the following species were measured every two days: Butorides virescens, Bubulcus ibis, Egretta thula, E. tricolor, E. caerulea, E. rufescens, Ardea alba and Nycticorax nycticorax. Logistic and Gompertz equations were adjusted to data using non-lineal regression models with adult values as the asymptote. For each species, the following were determined and recorded: growth rate, age at inflexion, instantaneous growth rates at each age interval, and time taken to reach $90 \%$ of adult size. Reported hatchling sizes were similar in other localities, with a variation coefficient ranging between 10-19\%. At hatch, each species exhibited differing sizes relative to adult values. In all cases, Gompertz equations were best fitted to explain more variance and lesser residuals. Rates of weight change and tarsus growth were alometrically related to the log of adult weight. Two main growth processes were identified: a physical extension in dimensions of each measurement reflecting inter-specific morphometric differences, and a lineal increase of the growth period from Green Heron to Great Egret. The Black-crowned Night Heron, Cattle Egret and Reddish Egret exhibited some unique measurement characteristics in comparison to the remaining members of the family. All results support the hypothesis that hypermorphosis, as the main evolutionary process in the microevolution of Ardeidae, is caused by a delayed final moment of growth. Rev. Biol. Trop. 59 (2): 771-787. Epub 2011 June 01.
\end{abstract}

Key words: egrets, herons, Ardea, Egretta, Butorides, Bubulcus, Nycticorax, Cuba.

Las dinámicas representadas en la forma y velocidad de las curvas de crecimiento de las diferentes estructuras corporales en las aves han sido moldeadas durante la evolución bajo numerosas presiones selectivas (Gill 1998). El crecimiento en aves ha recibido considerable atención, además de que sus implicaciones ecológicas han sido profundamente analizadas por Ricklefs (1968, 1975, 1979). Este proceso es receptor de numerosas fuerzas evolutivas y ecológicas contrapuestas que presionan simultáneamente. El desarrollo ontogenético de los pichones altriciales puede influir notablemente en su probabilidad de supervivencia
(Hochachka \& Smith 1991, Starck \& Ricklefs 1998), por tanto, existe una fuerte presión que promueve un rápido crecimiento hasta la independencia, lo que minimiza el tiempo de mayor vulnerabilidad por exposición a depredadores (Winkler \& Allen 1996, Monroes et al. 2002). Al mismo tiempo, se tiende a maximizar el tamaño en los pichones al independizarse, lo que se relaciona con el mayor desarrollo fisiológico y la habilidad de vuelo, lo cual aumenta la tasa de supervivencia (Ricklefs 1984, Linden et al. 1992), pero puede requerir de un mayor tiempo de desarrollo (Tinbergen \& Boerlijst 1990, Hochachka \& Smith 1991). 
El crecimiento en las aves generalmente se describe con una curva sigmoidea, en la cual un pequeño y lento crecimiento inicial es seguido de una fase de rápido crecimiento antes de abandonar el nido. Las informaciones de mayor interés en el crecimiento son la forma de la curva de crecimiento, su magnitud final o asíntota y la velocidad con la cual se alcanza (O’Connor 1984). Cada especie tiene una dinámica de crecimiento característico e inherente que puede ser analizada como parte de su estrategia reproductiva y puede reflejar su modo de desarrollo. La comparación de estos patrones brinda información valiosa acerca de las limitaciones ecológicas para la productividad y de las relaciones filogenéticas entre las especies. Las curvas de crecimiento pueden mostrar los momentos críticos del desarrollo postnatal de una especie (Massemin et al. 2002) lo que puede reflejar que los factores ambientales probablemente tengan mayores efectos.

Los ciconiformes son semialtriciales, y el desarrollo en muchas especies de este orden se caracteriza por un rápido crecimiento inicial del pico y las patas, especialmente los dedos, y una temprana habilidad para termorregular y moverse (Werschkul \& Jackson 1979). Kushlan (1977) describió el crecimiento diferencial de las partes corporales en Eudocimus albus y comentó el valor adaptativo de los diferentes patrones. La acelerada velocidad de crecimiento de los pichones representa una ventaja adaptativa que favorece crecimientos poblacionales acelerados, ya que reduce el período de nidificación, y permite aprovechar más efectivamente los pulsos de producción de alimentos en ambientes más inestables o impredecibles. Además, disminuye la probabilidad de muerte en el período más crítico del estadio de pichón y da a los padres la posibilidad de nidificar más de una vez durante una estación de cría, lo que aumenta la tasa de incremento de la población (Lack 1968). Este proceso permite disminuir la competencia con otras especies por recursos limitados en la época reproductiva, como los sitios de nidificación, el material para la construcción del nido, entre otros.
A pesar de la existencia de varios trabajos que describen este proceso, aún no puede decirse que la dinámica del crecimiento ha sido exhaustivamente caracterizada desde el punto de vista matemático en todas las especies de la familia, siendo los trabajos antiguos, enfocados en algunas especie puntuales y con poca cobertura geográfica. Las tasas de crecimiento son muy variables entre regiones y fechas y no pueden ser extrapolables de una región a otra. De cualquier forma, Freymann \& Schuchmann (2008) sugieren una revitalización de los estudios que recogen elementos básicos de la historia de vida, tal como las tasas de crecimiento postnatal en aves, especialmente en taxa tropicales.

El presente trabajo tiene como objetivo la caracterización de los patrones de crecimiento postnatal, en términos de velocidad y forma de las curvas de crecimiento, de los pichones de ocho especies de la familia Ardeidae: el Aguaitacaimán (Butorides virescens), la Garza Ganadera (Bubulcus ibis), la Garza de Rizos (Egretta thula), la Garza de Vientre Blanco (E. tricolor), la Garza Azul (E. caerulea), la Garza Rojiza (E.rufescens), el Garzón (Ardea alba) y el Guanabá de la Florida (Nycticorax nycticorax), durante las dos primeras semanas de vida en colonias de la ciénaga de Birama, Cuba.

\section{MATERIALES Y MÉTODOS}

El trabajo se realizó en la ciénaga de Birama, provincia Granma, en el área comprendida dentro del Refugio de Fauna "Delta del Cauto" $\left(20^{\circ} 28^{\prime} \mathrm{N}-77^{\circ} 10^{\prime} \mathrm{W}\right)$ en el período comprendido entre los años 1998 y 2006. La ciénaga de Birama es el segundo mayor humedal costero de Cuba y el Caribe, con un área de $570.5 \mathrm{~km}^{2}$ y se ubica en la desembocadura del río Cauto, el más caudaloso del país. Es una ciénaga de tipo deltaica que incluye un intrincado sistema de estuarios, lagunas, ciénagas y pantanos de elevado grado de preservación, declarada sitio Ramsar desde 2004 y que contiene dos áreas protegidas con la categoría de Refugio de Fauna (categoría IV de la UICN). 
En esta ciénaga destaca la laguna Las Playas, extenso cuerpo de aguas someras, con alrededor de $12 \mathrm{~km}^{2}$, que comunica con el mar a $11 \mathrm{~km}$ de su borde oriental. En medio y alrededor de esta se forman anualmente varias colonias reproductivas de aves acuáticas con un comportamiento metapoblacional bien establecido (Denis 2001). El presente trabajo se realizó en seis colonias de garzas: Cayo Norte, Wiso, La Güija, La Nueva, La Flora, La Salina y en un área de nidificación de aguaitacaimán, a los lados del canal de la camaronera.

Para caracterizar el crecimiento postnatal de los pichones, entre los años 1998 y 2004, se midieron, en días alternos durante dos semanas, el peso corporal (g), la longitud del pico (mm, desde la base superior de la ranfoteca hasta la punta) y la longitud del tarso ( $\mathrm{mm}$, desde la articulación doblada del tibio-tarso hasta las articulaciones de los dedos doblados hacia atrás). Por consiguiente, se emplearon en las mediciones balanzas de campo (pesola, $1 \mathrm{~g}$ de precisión) y un pie de rey ( $0.01 \mathrm{~mm}$ de precisión).

En los años 2005 y 2006 se siguió el método de Ricklefs \& White (1975) para construir curvas de crecimiento de los pichones de garza azul a partir de visitas breves, para lo cual de cada pichón se hicieron dos mediciones, separadas tres días entre sí. En total, se midieron 714 pichones: 97 de Aguaitacaimán (sigla empleada en las figuras: Ag), 217 de Garza Ganadera (GG), 151 de Garza de Rizos (GR), 137 de Garza de Vientre Blanco (GVB), 20 de Garza Rojiza (GRj), 43 de Guanabá de la Florida (GF), 27 de Garzón (Gz), 20 de Garza Azul (GA) y seis de Guanabá Real (GRl). El seguimiento de los pichones solo se realizó en las dos primeras semanas de vida, debido a que a edades mayores la movilidad de los pichones dificultaba su captura y manipulación sin producirles un estrés severo e introducir disturbio en las colonias, y aumentar la mortalidad.

Los nidos, previamente marcados, se visitaban diariamente para detectar las eclosiones, que fueron tomadas para determinar la edad de los pichones. El día de eclosión se tomó como edad uno. Cuando la eclosión no se detectaba directamente, se estimaba a partir de la edad de alguno de los hermanos del nido cuya eclosión hubiera sido observada, se asume un intervalo de dos días entre eclosiones, que es el valor medio de asincronía en la mayoría de las garzas (Maxwel \& Kale 1977, Custer et al. 1992). Los nidos que se encontraban con todos los pichones eclosionados, sin tener referencia alguna a su edad real, eran desestimados.

Si bien el crecimiento en las dos primeras semanas tiene un comportamiento cercano a una recta, no es adecuado aplicar un modelo de regresión lineal al no cumplirse los supuestos de estos métodos, en particular la independencia de los valores y la homogeneidad de varianza, además de que este método ignoraría las diferencias en valores finales teóricos del crecimiento en cada especie, que pueden influir notablemente en los estimados de constantes de crecimiento. Por estas razones se condujeron regresiones no lineales a los modelos teóricos de crecimiento sigmoideo más usados en este tipo de estudio.

Se utilizaron las ecuaciones logística y de Gompertz, que son las formas más usuales de describir el crecimiento en los animales, particularmente, en las aves (Ricklefs 1967). Inicialmente también se consideraron las ecuaciones de Von Bertalanffy y de Richards (Von Bertalanffy 1960), pero fueron desestimadas, en el primer caso por su pobre ajuste relativo $\mathrm{y}$ en el segundo caso por presentar mucha inestabilidad en su estimación en la regresión no lineal. Ratkowsky (1983) y Thornley \& Johnson (1990) ya habían advertido los sesgos y problemas prácticos asociados a estas ecuaciones de crecimiento, a pesar de la flexibilidad que ofrece y las nuevas variantes propuestas. Las diferencias entre las curvas utilizadas se establecen principalmente en la duración relativa de las etapas iniciales y finales del crecimiento y en el punto de inflexión, que en la curva Logística se asume que se encuentre en la mitad del crecimiento y en la de Gompertz en $1 /$ euler.

El ajuste no lineal se realizó por el método de Levenberg- Marquardt (combinado con el método de movimiento de patrones de HookeJeeves), y como medida del ajuste se tomó el 
porcentaje de varianza explicada por el modelo y la desviación estándar de los residuos, para seleccionar el modelo que más se acerca a los datos. Se comprobó el cumplimiento de las asunciones del modelo de regresión analizando la distribución de los residuos. Las diferencias en el crecimiento -usando estas ecuacionesse determinaron por la comparación de dos parámetros fundamentales: la constante de crecimiento (k) y la edad a la cual se alcanza el punto de inflexión $\left(\mathrm{t}_{i}\right)$. Para facilitar las comparaciones e interpretación de estos resultados, también se calculó el tiempo requerido para completar el $90 \%$ del crecimiento $\left(\mathrm{t}_{90}\right)$. La tasa de crecimiento instantáneo en cada edad fue calculada según la ecuación de Brody (1964) $\left(\mathrm{k}_{i}=\ln \mathrm{V}_{2} / \mathrm{V}_{1}\right.$; donde $\mathrm{V}$ : variable medida en los momentos uno y dos).

Existe muy poca información acerca del peso o las dimensiones en el momento de la independencia. En la Garza Azul la asíntota de la masa fue sugerida en un $25 \%$ inferior al valor adulto, y las del pico y tarso en valores entre 15-20\% inferiores a este (Werschkul 1979), pero en la Garza de Vientre Blanco, las tendencias sugieren un valor asintótico igual al adulto o ligeramente superior (Black et al. 1984). Ante esta incertidumbre, la asíntota superior fue asumida como equivalente al valor adulto promedio, debido a que incluirla como parámetro estimable en la ecuación puede producir valores biológicamente irreales, dado que el conjunto de datos está truncado en edades relativamente tempranas (Brisbin et al. 1987). A pesar de no haberse obtenido datos en etapas superiores de crecimiento, se consideró el uso de funciones sigmoideas, ya que al fijar su asíntota final en valores de peso que no provienen del propio crecimiento sino de valores con significación biológica (medias poblacionales de los adultos) se restringe la alta variabilidad potencial en los parámetros de forma y temporales del crecimiento.

Las medidas morfológicas empleadas como asíntotas del crecimiento para el presente trabajo, se tomaron de muestreos realizados en poblaciones del Sur del Jíbaro (SanctiSpiritus) y Amarillas (Matanzas), ubicadas a unos $200 \mathrm{~km}$ del área de estudio, obviando posibles variaciones geográficas (Cuadro 1). Como excepción, los de Garza Rojiza fueron tomados de la bibliografía (Hancock \& Kushlan 1984), al no existir registros previos para Cuba.

Los pesos relativos al nacer se compararon con una prueba de Kruskall-Wallis y una comparación múltiple no paramétrica de tendencias

\section{CUADRO 1}

Medidas de los adultos de ocho especies de garzas (Aves: Ardeidae) empleadas como asíntotas en el análisis del crecimiento

TABLE 1

Adult measurements in eight species of egrets and herons (Aves: Ardeidae) used as asymptote in growth analysis

\begin{tabular}{llccccc}
\multicolumn{1}{c}{ Especie } & \multicolumn{1}{c}{ Nombre científico } & Sigla & Peso (g) & Pico (mm) & Tarso (mm) & $\begin{array}{c}\text { Tiempo de } \\
\text { independencia }\end{array}$ \\
Aguaitacaimán & Butorides virescens & Ag & 182.4 & 60.7 & 54.0 & 35 \\
Garza Ganadera & Bubulcus ibis & GG & 334.1 & 55.4 & 83.2 & 45 \\
Garza de Rizos & Egretta thula & GR & 363.2 & 79.3 & 100.4 & 49 \\
Garza Azul & Egretta caerulea & GA & 399.4 & 78.7 & 93.4 & 35 \\
Garza de V. Blanco & E.tricolor & GVB & 410.5 & 96.8 & 101.3 & 59 \\
Garza Rojiza* & E.rufescens & GRj & 454.5 & 97.0 & 153.6 & 49 \\
Guanabá de la Florida & Nycticorax nycticorax & GF & 846.0 & 75.0 & 83.5 & 49 \\
Garzón & Ardea alba & Gz & 1004.2 & 113.0 & 152.0 & 51
\end{tabular}

Fuente: Acosta \& Mugica, com. pers.; Hancock \& Kushlan (1984) (*) / Source: Acosta \& Mugica, pers. com.; Hancock \& Kushlan (1984) $(*)$. 
centrales. Entonces se hizo una correlación de Spearman entre el logaritmo de los pesos y las tasas de crecimiento entre especies; para emplear los errores estándares como estimadores de precisión y el nivel de significación en las pruebas de hipótesis fue de 0.05 . El procesamiento estadístico se realizó con el programa Statistica ver. 8 (StatSoft 2007).

\section{RESULTADOS}

Todas las garzas son especies semialtriciales, nidícolas, y al eclosionar el pichón tiene un bajo grado de desarrollo (Cuadro 2). El tamaño de los pichones al nacer se relaciona directamente con el tamaño de los adultos de cada especie, posiblemente a través del efecto del tamaño del huevo. Ni en el Garzón ni en la Garza Azul se obtuvieron medidas directas de pichones recién nacidos, pero en el segundo caso el valor inicial fue estimado a partir del método para la obtención de curvas con visitas breves a las colonias, descrito por Ricklefs \& White (1975). Dos pichones recién nacidos de Guanabá Real (Nyctanassa violacea), especie no incluida en el estudio por el pequeño tamaño de muestra alcanzado, tuvieron las siguientes medidas: peso: 22 y $34.5 \mathrm{~g}$; tarso: 13.6 y $20.7 \mathrm{~mm}$; y tarso: 13.8 y $19.8 \mathrm{~mm}$.

El peso fue la medida más variable al nacer en todas las especies, con coeficientes de variación de entre 10-19\%. Las especies de mayor variabilidad en el peso al nacer fueron la Garza Rojiza (CV=18.9\%) y la Garza Ganadera $(\mathrm{CV}=18.6 \%)$ y la de peso más constante fue la Garza de Vientre Blanco ( $\mathrm{CV}=9.77 \%)$. El tarso al nacer fue menos variable que el peso, con valores entre 4-6\% en todas las especies, excepto en el Aguaitacaimán y en el Garzón donde la variabilidad fue de 9 y $10 \%$ respectivamente. El coeficiente de variación del pico al nacer varió alrededor del $8 \%$, siendo mínimo en el Garzón (4.7\%) y máximo en la Garza Rojiza (12\%).

\section{CUADRO 2}

Dimensiones de los pichones al nacer en ocho especies de garzas (Aves: Ardeidae) en la ciénaga de Birama, Cuba, entre 1998 y 2006

\section{TABLE 2}

Hatchling sizes in eight species of egrets and herons (Aves: Ardeidae) in Birama swamp, Cuba, between 1998 and 2006

\begin{tabular}{lccc}
\multicolumn{1}{c}{ Especie } & Peso $(\mathrm{g})$ & Pico $(\mathrm{mm})$ & Tarso $(\mathrm{mm})$ \\
Aguaitacaimán & $14.8 \pm 0.62(16)$ & $10.3 \pm 0.20(17)$ & $16.2 \pm 0.36(17)$ \\
& $(12.0-20.0)$ & $(8.8-11.8)$ & $(13.9-19.9)$ \\
Garza Ganadera & $20.8 \pm 0.57(46)$ & $12.2 \pm 0.14(47)$ & $18.3 \pm 0.15(44)$ \\
Garza de Rizos & $(14.0-29.0)$ & $(10.2-14.6)$ & $(16.4-20.2)$ \\
& $17.4 \pm 0.34(60)$ & $11.9 \pm 0.13(61)$ & $18.1 \pm 0.15(62)$ \\
Garza de Vientre Blanco & $(14.0-24.0)$ & $(9.6-14.6)$ & $(15.5-21.4)$ \\
Garza Azul* & $18.3 \pm 0.30(35)$ & $12.5 \pm 0.18(36)$ & $19.1 \pm 0.14(34)$ \\
Garza Rojiza & $(14.0-23.5)$ & $(10.2-15.3)$ & $(17.2-20.6)$ \\
& - & 12.1 & 19.0 \\
Guanabá de La Florida & $30.3 \pm 2.02(8)$ & $13.1 \pm 0.39(16)$ & $22.1 \pm 0.24(14)$ \\
Garzón & $(23.5-40.5)$ & $(9.1-14.8)$ & $(20.5-24.0)$ \\
& $38.0 \pm 6.39(5)$ & $15.1 \pm 0.23(4)$ & $21.0 \pm 0.77(5)$ \\
& $(28.0-62.0)$ & $(14.6-15.7)$ & $(19.5-23.7)$
\end{tabular}

Valores estimados por el método de Ricklefs y White (1975). Se utilizaron los promedios por nidada. Media \pm ES (n: no. de nidos), Min-Max. / Averages per nest were used. Mean \pm SE (n: no. of nests), Min-Max. 
Además de las diferencias absolutas al nacer, lógicas entre especies de tallas tan diferentes, al relativizar las medidas en función del valor adulto de cada especie se detectan diferencias significativas que reflejan variables grados de desarrollo en el momento de la eclosión. En el peso relativo al nacer se diferenciaron dos grupos (Kruskal-Wallis, $\mathrm{gl}=170, \mathrm{H}=92.06$, $\mathrm{p}<0.001$ ), uno formado por la Garza de Rizos, la Garza de Vientre Blanco y el Guanabá de la Florida, que eclosionaron con un peso inferior al 5\% del peso adulto (Fig. 1A). El Aguaitacaimán, el Guanabá de la Florida y la Garza Rojiza eclosionaron con pesos entre el 6 y el $8 \%$ del valor adulto. El tarso relativo al nacer mostró mayores diferencias relativas entre especies (Kruskal-Wallis, gl=183, H=153.40, $\mathrm{p}<0.001$ ), ya que la Garza de Vientre Blanco, la Garza Rojiza y el Garzón eclosionaron con cerca del $13 \%$ de tarso adulto mientras que en la Garza Ganadera y el Guanabá de la Florida este valor superó el 20\%. En la Garza Azul, a pesar de no medirse directamente, se estima que los pichones deben nacer también con un tarso bien desarrollado (20.4\% del adulto) (Fig. 1B).

El pico fue aún más variable en las proporciones al nacer entre especies (Kruskal-Wallis, $\mathrm{gl}=183, \mathrm{H}=153.40, \mathrm{p}<0.001)$, y se diferencian tres grupos (Fig. 1C). El Aguaitacaimán eclosionó con el pico muy desarrollado, de un $30 \%$ de valor adulto, seguido del Guanabá de la Florida (25\%) y la Garza Ganadera (22\%). La Garza Rojiza por el contrario eclosionó con un pico de solo el $14.4 \%$ del adulto, mientras que en el resto de las especies, esta dimensión al nacer se mantuvo entre el 16-18\%.

El crecimiento en las primeras dos semanas de vida tuvo un comportamiento exponencial, no muy alejado de una línea recta (Fig. 2). A diferencia de lo reportado en numerosos trabajos, posiblemente por efecto de considerar solo los valores de las primeras dos semanas de vida, la ecuación de Gompertz fue la que mejor ajuste alcanzó en todas las especies y variables, que juzga el ajuste por el porcentaje de varianza explicada en el modelo de regresión, superior al $95 \%$ en la mayoría de los casos (Cuadro 3). El modelo logístico también explicó un alto
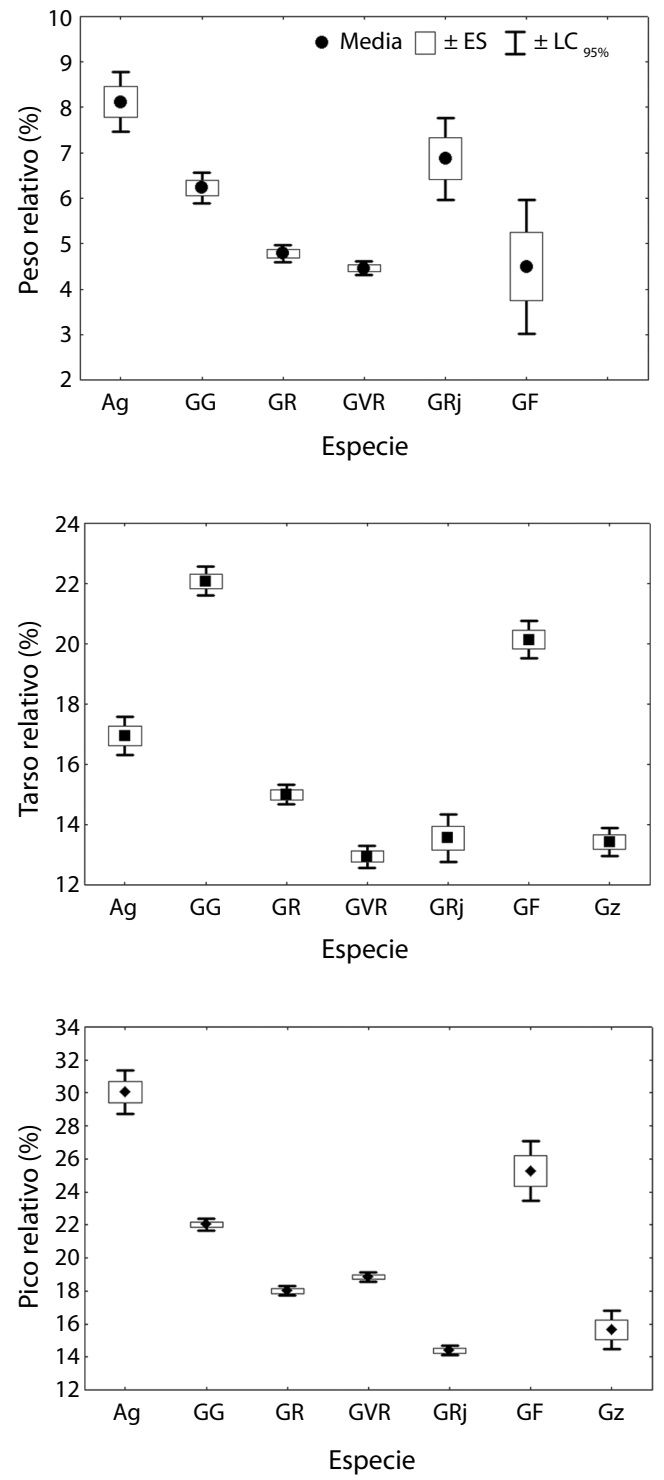

Fig. 1. Dimensiones de los pichones al eclosionar en ocho especies de garzas (Aves: Ardeidae), en la ciénaga de Birama, Cuba. Los valores están relativizados en función de las dimensiones de los adultos (Ag: B. virescens; GG: Bubulcus ibis, GR: Egretta thula, GVB: Egretta tricolor, GRj: E. rufescens, GF: Nycticorac nycticorax y Gz: E. alba).

Fig. 1. Relative hatchling sizes, in relation to adult size, in eight species of egrets and herons (Aves: Ardeidae), in Birama swamp, Cuba (Ag: B. virescens; GG: Bubulcus ibis, GR: Egretta thula, GVB: Egretta tricolor, GRj: E. rufescens, GF: Nycticorac nycticorax y Gz: E. alba). 


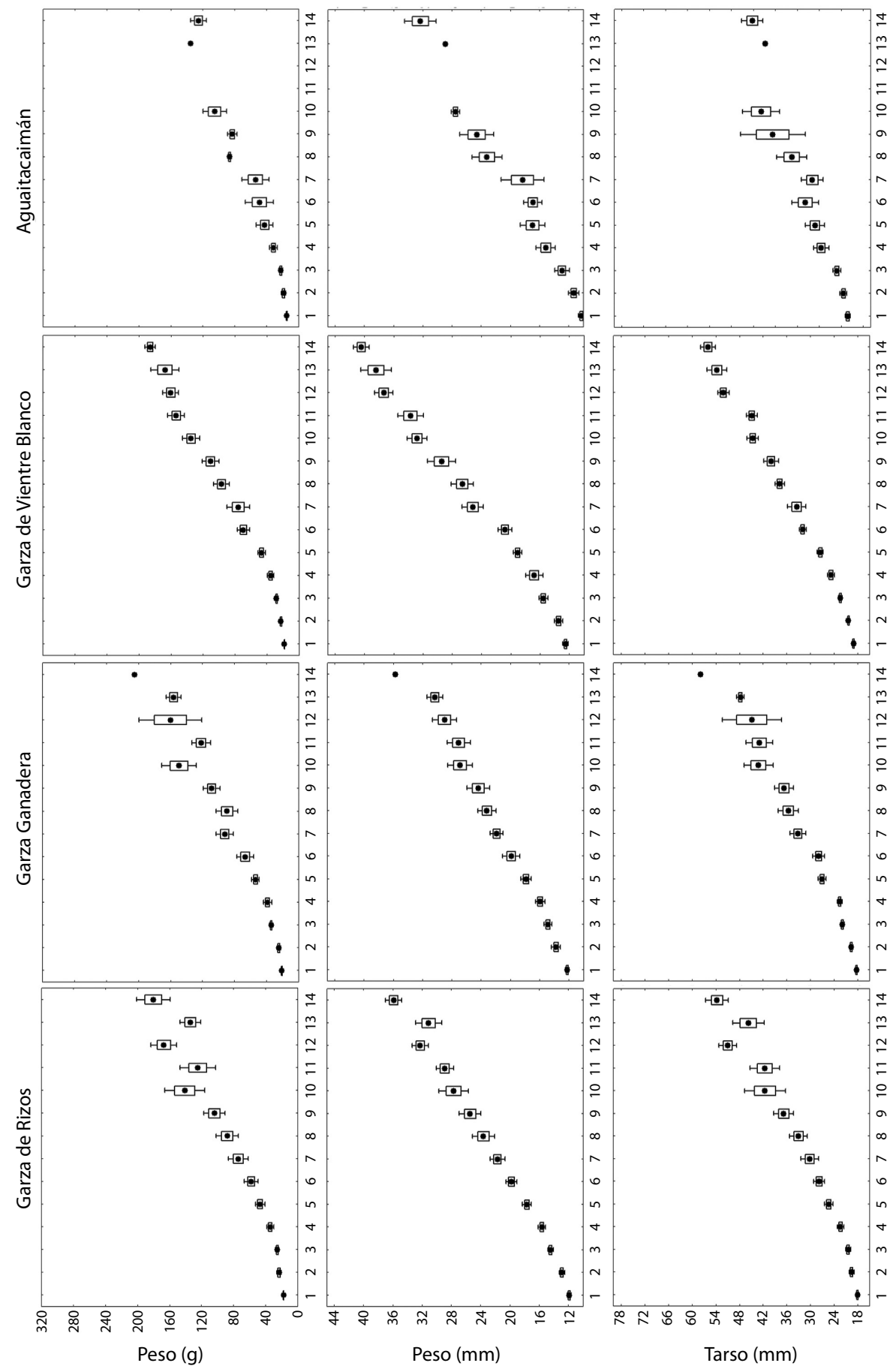

Fig. 2. Curvas de crecimiento del peso, pico y tarso en ocho especies de garzas (Aves: Ardeidae) durante las dos primeras semanas de vida, en la ciénaga de Birama, Cuba.

Fig. 2. Growth curves of weight, bill and tarsus in eight species of egrets and herons (Aves: Ardeidae) during the first two weeks in Birama swamp, Cuba. 
CUADRO 3

Estadísticos de ajuste de las ecuaciones de crecimiento Logística y de Gompertz en cada variable corporal, para ocho especies de garzas (Aves: Ardeidae)

TABLE 3

Fit statistics of Logistic and Gompertz' growth equations for each variable in eight species of egrets and herons (Aves: Ardeidae)

\begin{tabular}{|c|c|c|c|c|c|}
\hline \multirow{2}{*}{ Especie } & \multirow{2}{*}{ Variable } & \multicolumn{2}{|c|}{ Logística } & \multicolumn{2}{|c|}{ Gompertz } \\
\hline & & $\operatorname{Var}_{\text {Exp }}$ & $\mathrm{SD}_{\text {resid }}$ & $\operatorname{Var}_{\text {Exp }}$ & $\mathrm{SD}_{\text {resid }}$ \\
\hline \multirow[t]{3}{*}{ Aguaitacaimán } & Peso & 95.18 & 8.96 & 97.52 & 6.58 \\
\hline & Pico & 91.67 & 2.08 & 95.33 & 1.56 \\
\hline & Tarso & 90.29 & 2.78 & 93.87 & 2.23 \\
\hline \multirow[t]{3}{*}{ Garza Ganadera } & Peso & 94.29 & 13.73 & 95.77 & 11.89 \\
\hline & Pico & 96.19 & 1.35 & 97.71 & 1.05 \\
\hline & Tarso & 95.93 & 2.39 & 97.18 & 2.01 \\
\hline \multirow[t]{3}{*}{ Garza de Rizos } & Peso & 91.78 & 15.71 & 94.59 & 12.83 \\
\hline & Pico & 95.08 & 1.69 & 97.66 & 1.17 \\
\hline & Tarso & 95.03 & 2.62 & 97.18 & 1.99 \\
\hline \multirow{3}{*}{ Garza de Vientre Blanco } & Peso & 95.48 & 12.29 & 98.34 & 7.50 \\
\hline & Pico & 94.76 & 2.18 & 97.76 & 1.43 \\
\hline & Tarso & 96.38 & 2.36 & 98.67 & 1.44 \\
\hline \multirow[t]{2}{*}{ Garza Azul } & Pico & 98.33 & 2.23 & 99.66 & 1.05 \\
\hline & Tarso & 98.07 & 2.89 & 99.56 & 1.44 \\
\hline \multirow[t]{3}{*}{ Garza Rojiza } & Peso & 97.82 & 12.17 & 98.99 & 8.50 \\
\hline & Pico & 96.95 & 1.65 & 99.01 & 0.94 \\
\hline & Tarso & 97.27 & 2.40 & 99.27 & 1.25 \\
\hline \multirow[t]{3}{*}{ Guanabá de la Florida } & Peso & 77.62 & 19.46 & 79.88 & 18.47 \\
\hline & Pico & 95.23 & 1.42 & 98.04 & 0.91 \\
\hline & Tarso & 96.83 & 1.94 & 97.04 & 1.89 \\
\hline \multirow[t]{2}{*}{ Garzón } & Pico & 91.85 & 2.14 & 95.39 & 1.62 \\
\hline & Tarso & 96.19 & 2.03 & 98.29 & 1.37 \\
\hline
\end{tabular}

$\operatorname{Var}_{\text {Exp }}$ : explained variances $(\%) ; \mathrm{SD}_{\text {resid }}$ : Standard deviation of los regression residuals.

porcentaje de la varianza, superior al $90 \%$, pero la desviación estándar de los residuos de la regresión, que es una medida más directa de la calidad del ajuste, es mucho mayor en este modelo en relación al de Gompertz. En todas las especies el mejor ajuste se logró en el pico, excepto para el Garzón. En ambos modelos, solo el peso del Guanabá de La Florida no alcanzó significación estadística, posiblemente por efecto del pequeño tamaño de muestra y su alta variabilidad, por lo que esos resultados no son concluyentes.

En las dos ecuaciones utilizadas, el crecimiento es descrito por dos parámetros: la constante de crecimiento $(\mathrm{k})$ y un parámetro relacionado a la edad en el punto de inflexión de la curva (b) (Cuadro 4). Ambos parámetros no son comparables entre curvas (O'Connor 1984), pero sí entre especies descritas por la misma curva.

Las tasas de crecimiento del peso y del tarso estuvieron alométricamente relacionadas con el peso corporal de las especies (Fig. 3. A, C), igual a lo encontrado por Ricklefs et al. (1998). La correlación de Spearman detectó asociación significativa entre el logaritmo del peso y la constante de crecimiento del peso $\left(r_{s}=-0.738, p<0.05\right)$ y del tarso si se excluye 
CUADRO 4

Parámetros estimados por la regresión no lineal para las ecuaciones Logística y de Gompertz para modelar el crecimiento de cada variable corporal, en ocho especies de garzas (Aves: Ardeidae)

TABLE 4

Parameters of the Logistic and Gompertz' equations, estimated by non lineal regression, used to model the growth of each variable in eight species of egrets and herons (Aves: Ardeidae)

\begin{tabular}{|c|c|c|c|c|c|}
\hline \multirow{2}{*}{ Especie } & \multirow{2}{*}{ Variable } & \multicolumn{2}{|c|}{ Logística } & \multicolumn{2}{|c|}{ Gompertz } \\
\hline & & $\mathrm{b}$ & $\mathrm{k}_{L}$ & $\mathrm{~b}$ & $\mathrm{k}_{G}$ \\
\hline \multirow[t]{3}{*}{ Aguaitacaimán } & Peso & $24.7 \pm 8.9$ & $0.314 \pm 0.039$ & $1.63 \pm 0.13$ & $0.200 \pm 0.016$ \\
\hline & Pico & $19.78 \pm 5.86$ & $0.203 \pm 0.027$ & $1.31 \pm 0.09$ & $0.111 \pm 0.009$ \\
\hline & Tarso & $13.99 \pm 5.07$ & $0.269 \pm 0.042$ & $1.32 \pm 0.23$ & $0.177 \pm 0.031$ \\
\hline \multirow[t]{3}{*}{ Garza Ganadera } & Peso & $28.41 \pm 9.56$ & $0.258 \pm 0.030$ & $1.53 \pm 0.13$ & $0.144 \pm 0.013$ \\
\hline & Pico & $20.69 \pm 4.61$ & $0.222 \pm 0.020$ & $1.34 \pm 0.07$ & $0.122 \pm 0.007$ \\
\hline & Tarso & $25.17 \pm 6.34$ & $0.252 \pm 0.023$ & $1.47 \pm 0.09$ & $0.142 \pm 0.009$ \\
\hline \multirow[t]{3}{*}{ Garza de Rizos } & Peso & $29.07 \pm 9.65$ & $0.237 \pm 0.029$ & $1.51 \pm 0.14$ & $0.128 \pm 0.013$ \\
\hline & Pico & $27.40 \pm 5.64$ & $0.198 \pm 0.018$ & $1.39 \pm 0.06$ & $0.097 \pm 0.005$ \\
\hline & Tarso & $31.33 \pm 8.93$ & $0.232 \pm 0.024$ & $1.50 \pm 0.08$ & $0.120 \pm 0.008$ \\
\hline \multirow[t]{3}{*}{ Garza de Vientre Blanco } & Peso & $30.68 \pm 8.21$ & $0.235 \pm 0.023$ & $1.52 \pm 0.07$ & $0.125 \pm 0.007$ \\
\hline & Pico & $28.65 \pm 5.40$ & $0.201 \pm 0.017$ & $1.41 \pm 0.06$ & $0.099 \pm 0.005$ \\
\hline & Tarso & $25.05 \pm 5.37$ & $0.227 \pm 0.019$ & $1.43 \pm 0.06$ & $0.122 \pm 0.005$ \\
\hline \multirow[t]{2}{*}{ Garza Azul } & Pico & $28.11 \pm 7.47$ & $0.205 \pm 0.017$ & $1.73 \pm 0.06$ & $0.132 \pm 0.004$ \\
\hline & Tarso & $18.72 \pm 3.81$ & $0.196 \pm 0.014$ & $1.53 \pm 0.06$ & $0.132 \pm 0.004$ \\
\hline \multirow[t]{3}{*}{ Garza Rojiza } & Peso & $45.37 \pm 15.49$ & $0.302 \pm 0.030$ & $1.80 \pm 10.08$ & $0.170 \pm 0.008$ \\
\hline & Pico & $33.41 \pm 6.48$ & $0.213 \pm 0.016$ & $1.47 \pm 0.04$ & $0.103 \pm 0.004$ \\
\hline & Tarso & $42.92 \pm 7.84$ & $0.227 \pm 0.015$ & $1.56 \pm 0.04$ & $0.107 \pm 0.004$ \\
\hline \multirow[t]{3}{*}{ Guanabá de La Florida } & Peso & $80.20 \pm 44.57$ & $0.285 \pm 0.071$ & $1.60 \pm 0.18$ & $0.101 \pm 0.024$ \\
\hline & Pico & $37.44 \pm 9.56$ & $0.235 \pm 0.023$ & $1.51 \pm 0.06$ & $0.113 \pm 0.006$ \\
\hline & Tarso & $55.00 \pm 20.51$ & $0.327 \pm 0.035$ & $1.83 \pm 0.14$ & $0.176 \pm 0.015$ \\
\hline \multirow[t]{2}{*}{ Garzón } & Pico & $33.86 \pm 7.48$ & $0.173 \pm 0.019$ & $1.40 \pm 0.072$ & $0.076 \pm 0.006$ \\
\hline & Tarso & $43.66 \pm 9.24$ & $0.198 \pm 0.017$ & $1.49 \pm 0.05$ & $0.085 \pm 0.004$ \\
\hline
\end{tabular}

Estimado \pm ES / Estimate \pm ES

el Guanabá de la Florida $\left(\mathrm{r}_{\mathrm{s}}=-0.892, \mathrm{p}<0.05\right)$. Esta especie tiene una tasa de crecimiento del tarso muy superior a la esperable por su tamaño corporal, probablemente relacionada a los hábitos alimentarios y la conducta de los pichones. La tasa de crecimiento del pico fue mucho más variable en este grupo de aves, no detectándose asociación significativa con el peso corporal $\left(\mathrm{r}_{\mathrm{s}}=-0.381, \mathrm{p}=0.36\right)$ (Fig. 3. B). En la Garza Ganadera, el Guanabá de La Florida y la Garza Azul fueron superiores las tasas de crecimiento relativas al peso adulto.
El crecimiento según una dinámica sigmoidea implica una variación no lineal en las tasas de crecimiento instantáneas por edad $\left(\mathrm{k}_{i}\right)$, que presentaron dinámicas diferentes entre estructuras corporales (Fig. 4). El peso corporal tuvo su máxima $\mathrm{k}_{i}$ a los cinco días en el Aguaitacaimán, la Garza Ganadera, la Garza de Rizos y la Garza Rojiza, y a los seis días en la Garza de Vientre Blanco y la Garza Rojiza. En el Guanabá de La Florida esta se alcanzó a los siete días. Para el tarso el punto de máxima tasa instantánea de crecimiento se alcanzó entre los 

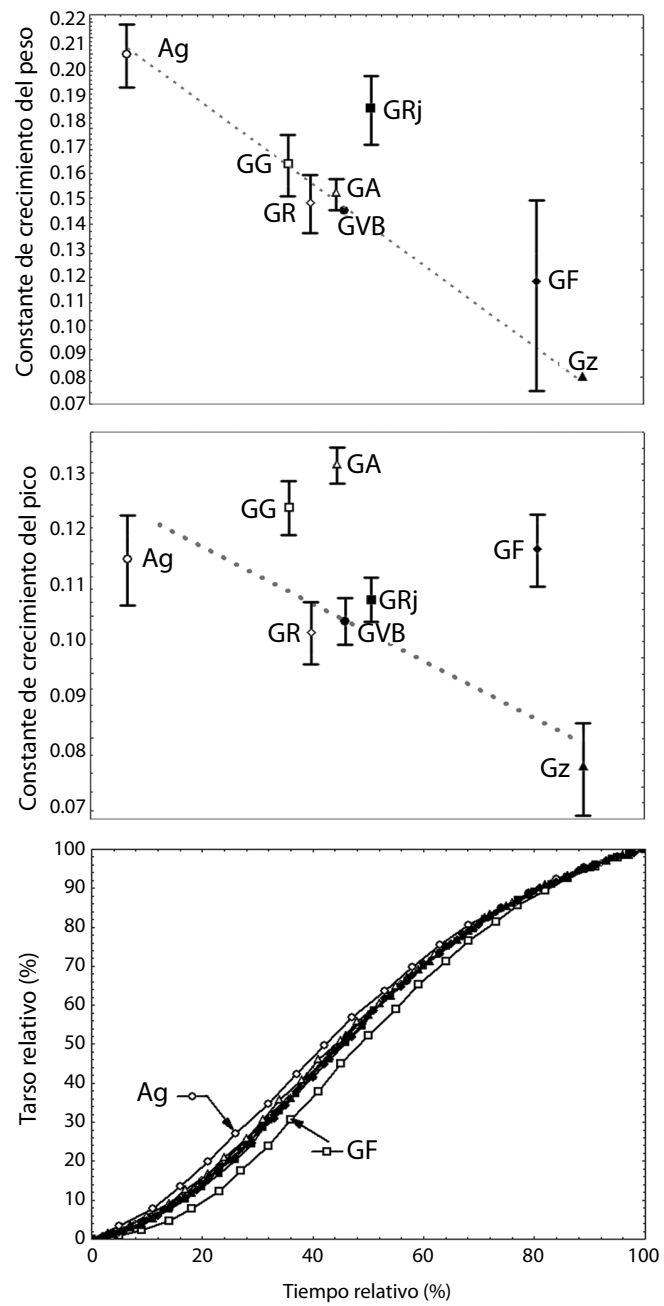

Fig. 3. Relaciones alométricas entre las constantes de crecimiento del pico y el tarso en ocho especies de garzas (Aves: Ardeidae) (ordenadas en escala logarítmica, las barras representan el error estándar del estimado de $\mathrm{k}$ por la regresión no lineal) (Ag: B. virescens; GG: Bubulcus ibis, GR: Egretta thula, GVB: Egretta tricolor, GRj: E. rufescens, GF: Nycticorac nycticorax y Gz: E. alba).

Fig. 3. Allometric relationship between growth rate of bill and tarsus in eight species of egrets and herons (Aves: Ardeidae) (ordinate in log scale, bars represent the standard error of the estimate by non lineal regression) (Ag: B. virescens; GG: Bubulcus ibis, GR: Egretta thula, GVB: Egretta tricolor, GRj: E. rufescens, GF: Nycticorac nycticorax y Gz: E. alba).

siete y ocho días en la mayoría de las especies. Los máximos valores se alcanzaron los días nueve y diez en el Garzón y la Garza Rojiza, respectivamente, y el mínimo valor fue del Aguaitacaimán, en el cual fue a los cinco días de eclosionado. El pico tuvo un crecimiento más lento, alcanzando la máxima tasa entre los siete y ocho días en la mayoría de las especies, excepto en el Guanabá de La Florida a los nueve días y en el Garzón a los diez días.

El crecimiento, sin embargo, no solo es suficientemente caracterizado por la velocidad de incremento de la estructura, sino que es importante analizar la duración del periodo de crecimiento. El estudio de los pichones hasta su total independencia es logísticamente difícil en este grupo, ya que a partir de los 14 días los pichones son muy móviles y escapan del nido al aproximarse el investigador y su persecución y captura pueden producir efectos negativos. Por esa razón, a partir de los parámetros de la curva de crecimiento se calculó el $\mathrm{T}_{90}$, que es el tiempo que demoraría el pichón en alcanzar, siguiendo esa dinámica, el $90 \%$ del valor adulto de la estructura analizada. Todas las especies alcanzaron ese desarrollo antes de la edad de independencia, señalada en la bibliografía para cada una.

A los 19 y 20 días los pichones de Aguaitacaimán alcanzan el $90 \%$ del peso y del tarso adultos, respectivamente, demorando otros 12 días para el pico, y varios días después se hacen independientes. La Garza Ganadera es la otra especie de más rápido desarrollo, ya que alcanza el $90 \%$ del peso y del tarso adultos en 26 días, y el pico en 30 días. A pesar de ello permanecen en las cercanías del nido, dependiendo de los padres por otras dos semanas. Los pichones de guanabáes en 23 días ya tienen el $90 \%$ del tarso adulto, pero demoran 33 y 38 días en alcanzar esta proporción del pico y del peso, respectivamente. En las garzas medianas -Garza de Rizos, Garza de Vientre Blanco y Garza Azul- entre los 30 y 37 días logran el $90 \%$ del peso, tarso y pico adultos, a pesar de que dependen por mucho más tiempo de los padres. La Garza Rojiza es notable la rapidez de crecimiento del peso a pesar de que el pico y el tarso demoran casi lo mismo o menos, que en las demás garzas medianas. La especie de crecimiento más lento y extendido es el 


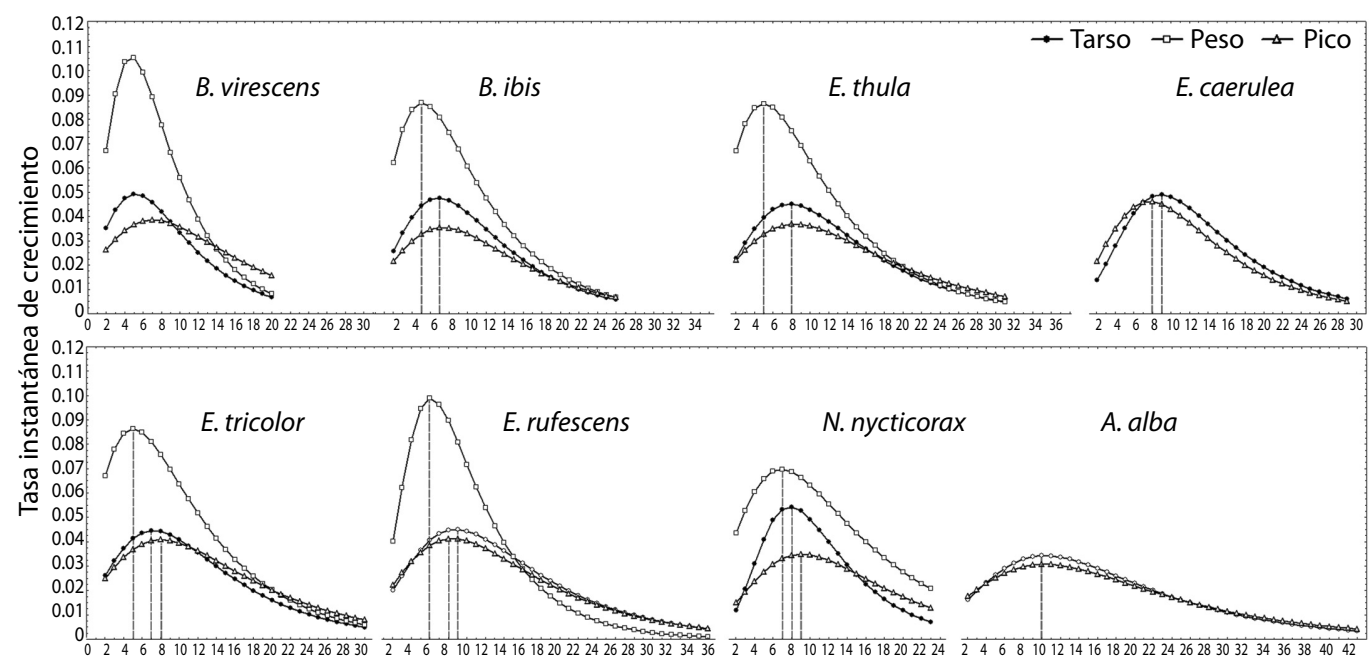

Fig. 4. Tasas instantáneas de crecimiento del peso, pico y tarso en ocho especies de garzas (Aves: Ardeidae) obtenidas de forma teórica a partir de las ecuaciones ajustadas al crecimiento durante las dos primeras semanas de vida y extrapoladas hasta que se alcanza el $90 \%$ del peso adulto.

Fig. 4. Instant Growth Rate of weight, bill and tarsus in eight species of egrets and herons (Aves: Ardeidae) theoretically estimated from adjusted equations to the first two weeks of growth, extrapolated to $90 \%$ of adult values.

Garzón, de la cual no existen datos suficientes de peso corporal pero que demora 44 y 48 días en alcanzar el $90 \%$ del tarso y del pico adulto, respectivamente, para unos días después abandonar a los parentales.

El análisis comparativo de las dinámicas de crecimiento del tarso, como estructura representativa de particular importancia ecológica en este grupo de especies, que tiene en cuenta simultáneamente las velocidades, duraciones y valores finales del crecimiento, muestra una tendencia interespecífica muy bien definida (Fig. 5). En el proceso de crecimiento se identifican dos procesos fundamentales: una extensión física de la estructura, que se refleja en las diferencias físicas entre las especies, con un alargamiento de la duración del periodo de crecimiento entre el Aguaitacaimán y el Garzón. La extensión temporal del proceso se relaciona linealmente con el incremento en la estructura, y se aleja de esta tendencia solo la Garza Rojiza. La representación de las dinámicas de crecimiento del tarso, que elimina las diferencias absolutas de talla y reescala el eje temporal a porcentaje del $\mathrm{T}_{90}$, superpone casi perfectamente las curvas (Fig. 6), y resalta solo los extremos, representados por el Aguaitacaimán y el Guanabá de La Florida.

\section{DISCUSIÓN}

El análisis del crecimiento postnatal en grupos de especies relacionadas puede arrojar luz sobre los patrones evolutivos que siguió el grupo durante su evolución. En el caso de las garzas, este fenómeno ha sido estudiado de forma reiterada, sin embargo, aún no se tiene una integración consistente de los resultados obtenidos en diferentes momentos y lugares. Los pesos de los pichones recién eclosionados encontrados en el presente trabajo son similares a los reportados en otras localidades, en la Garza de Rizos (Parsons \& Master 2000), la Garza Ganadera (Telfair 1983) y la Garza Azul (McVaugh 1972). El tarso en los pichones recién eclosionados, sin embargo, es superior al reportado por Telfair (1983) (13.1$16.4 \mathrm{~mm}, \mathrm{n}=8)$. También el peso del pichón 

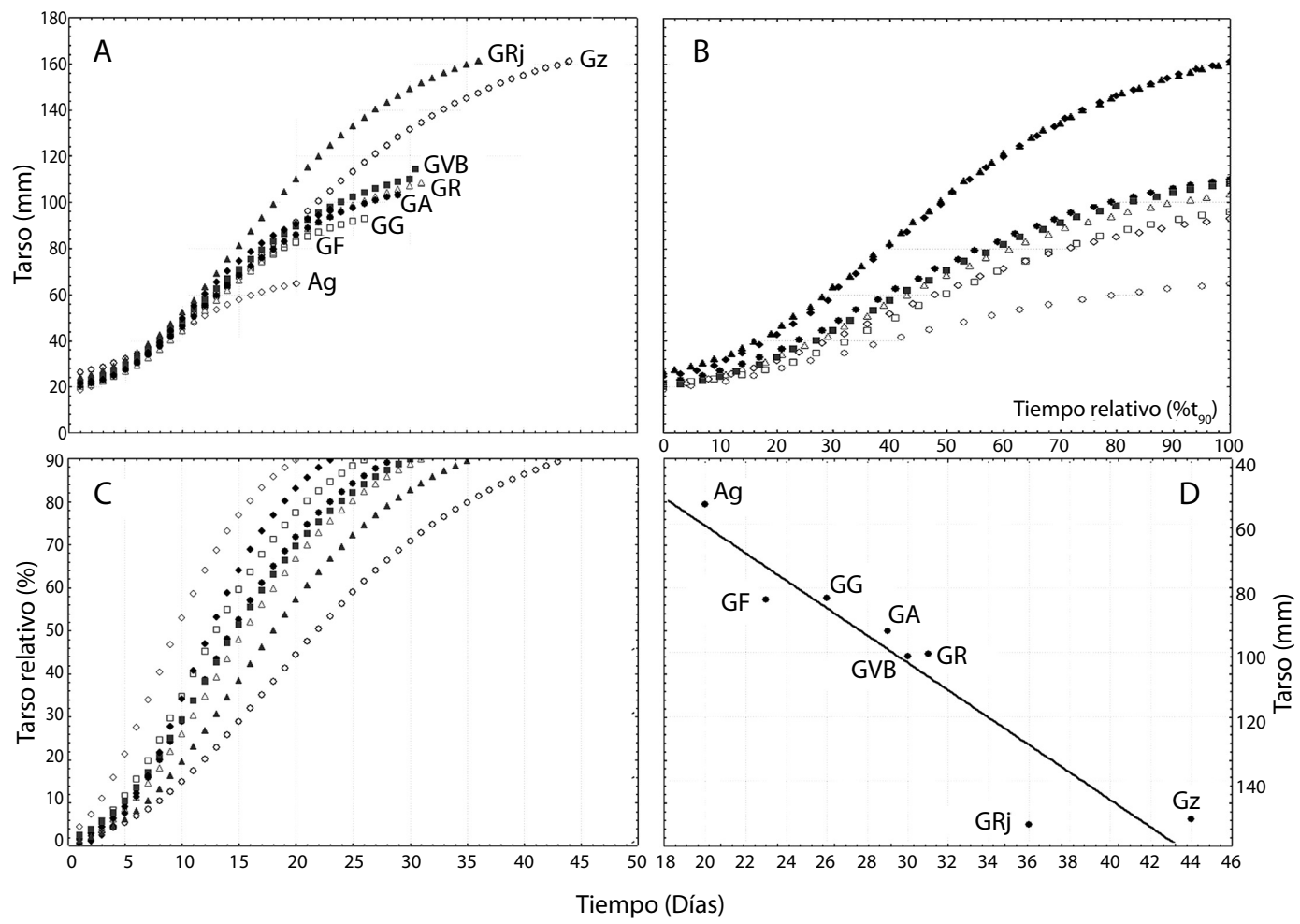

Fig. 5. Tendencias en la duración y el momento final del crecimiento entre ocho especies de garzas (Aves: Ardeidae). A: Curvas de crecimiento absoluto, B: Curvas de crecimiento con el eje del tiempo relativizado, C: Curvas de crecimiento con el eje del tarso relativizado, D: Tendencia general en la duración y final del crecimiento del tarso (Ag: B. virescens; GG: Bubulcus ibis, GR: Egretta thula, GVB: Egretta tricolor, GRj: E. rufescens, GF: Nycticorac nycticorax y Gz: E. alba).

Fig. 5. Trends in duration and final time of growth in eight species of egrets and herons (Aves: Ardeidae). A: Absolute growth curves, B: Growth curves with relative temporal axis, C: Growth curves with relative tarsus axis, D: General trends in duration and ending of growth of tarsus (Ag: B. virescens; GG: Bubulcus ibis, GR: Egretta thula, GVB: Egretta tricolor, GRj: E. rufescens, GF: Nycticorac nycticorax y Gz: E. alba).

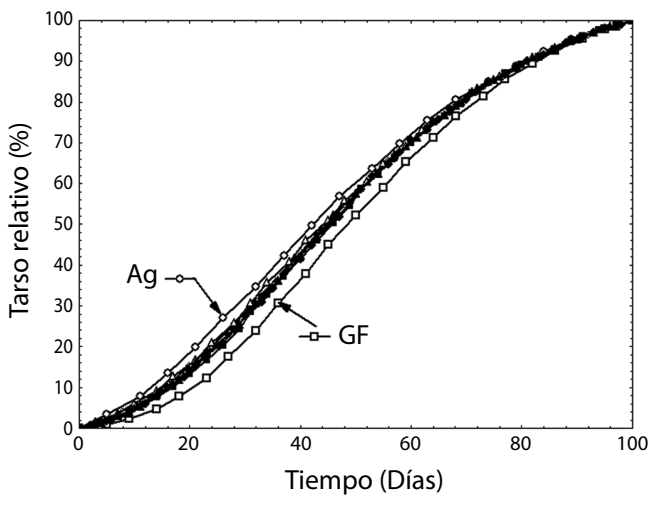

Fig. 6. Similitud funcional en la dinámica de crecimiento del tarso en ocho especies de garzas (Aves: Ardeidae), al estandarizarlas en dimensiones absolutas y en la duración del crecimiento. Las especies más alejadas son el Aguaitacaimán (Ag) y el Guanabá de La Florida (GF).

Fig. 6. Functional similarities in growth dynamics of tarsus in eight species of egrets and herons (Aves: Ardeidae), once eliminated differences in absolute dimensions and standardizing to growth duration. Outer species are Green Heron $(\mathrm{Ag})$ and Black-crowned Night Heron (GF). 
recién eclosionado citado por Davis (1993) para el Guanabá de La Florida fue inferior al encontrado en la ciénaga de Birama (24.2 g). Los valores recogidos por McVaugh (1972) en el Garzón para los días 1, 7 y 14 son ligeramente menores en todas las variables que los del presente trabajo.

La Garza Ganadera ha sido una de las especies más estudiadas en todos los sentidos, en la familia Ardeidae. Los pichones eclosionan con aproximadamente el $6 \%$ del peso adulto, pero a los 14 días ya han alcanzado casi el $70 \%$ de este valor, lo que coincide con lo encontrado por Siegfried (1972). En esta especie se ha descrito un pequeño pico en el crecimiento a los seis días, el cual coincide con la transición de plumón a plumas verdaderas, y otro entre los nueve y 12 días, que concuerda con el pico del consumo energético, valor superior al calculado en el presente estudio, donde la máxima tasa de crecimiento instantáneo se calcula entre cinco y ocho días. A los 11 días de eclosionados los pichones han alcanzado más del $40 \%$ del peso del adulto, lo que coincide con lo planteado por varios autores (Hudson et al. 1974, Dunn 1975, Telfair 1983). A partir de los 13 días el plumaje se desarrolla, y con la endotermia, disminuye el consumo de energía y la tasa se crecimiento (Telfair 1994).

En la Garza Ganadera el crecimiento sigmoideo fue representado por Telfair (1983) a través de un polinomios de tercer orden y encontró que el crecimiento más acelerado era el del pico, seguido del peso y del tarso. Otros trabajos han ajustado el crecimiento de cada estructura a diferentes ecuaciones (Siegfried 1973, Hudson et al. 1974, Telfair 1983). La tasa de crecimiento del peso encontrada en la ciénaga de Birama, según la ecuación de Gompertz, es similar a la descrita por Siegfried (1973) para Sudáfrica $\left(\mathrm{k}_{G}=0.148\right)$, y superior a los obtenidos por Werding (1970) en Madagascar $\left(\mathrm{k}_{G}=0.103\right)$ y por Ruiz (1981) en España $\left(\mathrm{k}_{G}=0.115\right)$. Sin embargo, estas diferencias pueden deberse al empleo de diferentes pesos como asíntotas del crecimiento. En la Garza de Vientre Blanco, y la Garza de Rizos los valores promedios del pico y el tarso dados por
Frederick (1997), McVaught (1972) y Palmer (1962), son muy semejantes a los encontrados en este trabajo.

La curva de Gompertz ha sido mencionada como la de mejor ajuste para describir el crecimiento de especies precociales o intermedias (Shanholtzer 1972). Ricklefs (1968) también encontró que el crecimiento en peso en tres especies de garzas era mejor descrito por la ecuación de Gompertz y para la Garza Ganadera, Ruiz (1981) encuentra un crecimiento de este tipo en las tres variables corporales analizadas. Según este último autor la tendencia a adoptar el crecimiento tipo Gompertz o von Bertalanffy obedece a la necesidad de minimizar el requerimiento energético en fases avanzadas del desarrollo cuando a las necesidades del crecimiento se adicionan las de otras actividades como la locomoción. También puede obedecer a la ventaja de tener un periodo de crecimiento inicial más corto, que reduzca la susceptibilidad a la depredación en las primeras etapas del desarrollo.

La acelerada velocidad de crecimiento de los pichones representa una ventaja adaptativa que favorece rápidos crecimientos poblacionales al reducir el período de nidificación, y permitir un aprovechamiento más efectivo de los pulsos de producción de alimentos en ambientes inestables o impredecibles como los humedales. Además, disminuye la probabilidad de muerte en el período más crítico del estadio de pichón y da a los padres la posibilidad de nidificar más de una vez durante una estación de cría, lo que aumenta la tasa de incremento de la población (Lack 1968). Este último factor permite disminuir la competencia por recursos limitados en la época reproductiva, como los sitios de nidificación, el material para la construcción del nido, etc.

Custer \& Peterson (1991), al comparar la velocidad de crecimiento del peso del Guanabá de la Florida, la Garza de Rizos y el Garzón encontraron diferencias significativas en la pendiente de la recta entre las tres especies. Entonces, obtuvieron que en el Guanabá de la Florida el peso se incrementa más rápidamente que el del Garzón que presenta mayor talla y 
peso corporal de adulto, al igual que ocurre en los resultados presentados con la Garza Ganadera y el Aguaitacaimán. Sin embargo, en el trabajo anterior, los autores asumieron linealidad en el crecimiento y además, realizaron las comparaciones utilizando las medidas absolutas y no las relativas, por lo que no tuvieron en cuenta las diferencias en tallas entre las especie, que en ausencia de diferencias notables en duración del período de preindependencia, influyen en la velocidad de crecimiento.

Las tasas de crecimiento calculadas en este trabajo son difíciles de comparar con valores previos publicados dada la diversidad de métodos empleados en su estimación. En la Garza Azul, el 90\% del crecimiento del pico y del tarso se alcanzó en fechas similares a las descritas por Werschkul (1979) (31.8 y 28.8 días, respectivamente). El $90 \%$ del crecimiento del peso, reportado por este autor se alcanzó a los 17.2 días. Un factor importante a tener en cuenta en estos estudios es la utilización de diferentes asíntotas para el cálculo de las velocidades de crecimiento ya que este está altamente correlacionado con la tasa de crecimiento (Ricklefs 1968), de ahí que las variaciones en la talla adulta o el dimorfismo sexual puedan influir en el tipo de ajuste encontrado en una especie determinada. Esto puede explicar en parte las diferencias encontradas con otros reportes de la literatura.

Starck \& Ricklefs (1998), a partir de recopilaciones bibliográficas, brindan una lista de parámetros del crecimiento de 557 especies, entre ellas ocho de la familia Ardeidae. Las tasas de crecimiento brindadas para el Aguaitacaimán y la Garza Ganadera fueron inferiores a los valores calculados en el presente trabajo (Ag: $\mathrm{k}_{G}=0.135 \mathrm{y} \mathrm{k}_{G}=0.149$, asíntota $200 \mathrm{~g}$; GG: $\mathrm{k}_{L}=0.210$; asíntota $338 \mathrm{~g} \mathrm{y} \mathrm{k}_{L}=0.231$; asíntota $267 \mathrm{~g} ; \mathrm{k}_{G}$ : según Gompertz, $\mathrm{k}_{L}$ : según Logística). En la Garza Azul, sin embargo, fueron superiores $\left(\mathrm{k}_{L}=0.258\right)$ posiblemente por haber usado asíntotas más bajas $(300 \mathrm{~g})$.

Las diferencias en la velocidad de crecimiento de las partes corporales dentro de cada especie tiene funciones ecológicas y ha sido descrita en varias especies (Kushlan 1977,
Werschkul 1979, Ruiz 1981, González-Martín et al. 1992). El crecimiento acelerado de las estructuras locomotoras en la Garza Azul, según Werschkul (1979), tiene un significado adaptativo relacionado con la relativa precocidad de los pichones, y les facilita trepar, asirse y caminar entre las ramas desde las primeras semanas de edad. Esto les permite mantenerse seguros cerca del nido o moverse en el árbol y en la tierra para escapar de los depredadores. La habilidad temprana para moverse puede ser una adaptación defensiva que "sustituye" la protección relativa proporcionada por la vida colonial en las zancudas, por ello las especies solitarias alcanzan mayores tasas de crecimiento del tarso. A esta conclusión también arribaron Cubo et al. (2000) al analizar el crecimiento de Ixobrychus minutus y Ardeola ralloides.

El incremento rápido del peso es típico de las especies altriciales que permanecen bastante tiempo en el nido después de la eclosión y son alimentadas por los padres, por lo que toman la mayor parte de la energía para el crecimiento y el incremento de la biomasa antes de desarrollar las funciones maduras, como plantea Ricklefs (1979) que ocurre con el Estornino Europeo. El crecimiento comparativamente más lento del pico puede estar relacionado con el gradual incremento de la habilidad para el forrajeo.

La relación lineal directa entre la extensión temporal del proceso de crecimiento entre las especies de garzas estudiadas con la magnitud del incremento en la estructura corporal sugiere la hipótesis de que el cambio fundamental en la microevolución del tarso en la familia Ardeidae fue una hipermorfosis causada por el retardo en el momento final del crecimiento, que al extenderse por más tiempo produce las estructuras relativamente incrementadas. Esta hipótesis es apoyada, además, por la similitud funcional de las dinámicas de crecimiento una vez eliminadas las diferencias temporales y de asíntota.

\section{AGRADECIMIENTOS}

El trabajo de campo fue posible gracias al apoyo de la Empresa Nacional para la 
Protección de la Flora y la Fauna, y a los trabajadores del Refugio de Fauna Delta del Cauto; en especial, a Omar Labrada por su inestimable apoyo logístico. Se agradece a Ideawild por proveer equipamiento e instrumentos de medición y a un gran número de estudiantes de la carrera de Biología que participaron en la toma de datos, especialmente a Patricia Rodríguez Casariego.

\section{RESUMEN}

El crecimiento en aves forma parte de las estrategias reproductivas y su implicación ecológica ha sido profundamente debatida. En este trabajo se describe el crecimiento del pico, peso y tarso de ocho garzas (Aves: Ardeidae) en la ciénaga de Birama, Cuba. Para ello, entre 1998-2006, se midieron en días alternos un total de 714 pichones de Butorides virescens, Bubulcus ibis, Egretta thula, E. tricolor, E. caerulea, E. rufescens, Ardea alba y Nycticorax nycticorax, durante sus dos primeras semanas de vida. Se hicieron ajustes a las curvas Logística y de Gompertz y se usó regresiones no lineales y valores adultos como asíntotas, además se determinaron las variables que describen el crecimiento. El tamaño de los pichones al eclosionar fue similar al encontrado en otras localidades, con CV entre $10-19 \%$ y diferencias relativas al tamaño adulto. Las ecuaciones de Gompertz tuvieron menos residuos en todos los casos. Entonces, se identificaron dos procesos fundamentales de extensión en el crecimiento de cada estructura: uno físico y otro de duración, entre $B$. virescens y E. alba. Nycticorax, B. ibis y E. rufescens mostraron variaciones que los diferenciaron del resto. Los resultados apoyan la hipótesis de la microevolución de Ardeidae por hipermorfosis, por un retardo en el momento final del crecimiento.

Palabras clave: garzas, Ardea, Egretta, Butorides, Bubulcus, Nycticorax, Cuba.

\section{REFERENCIAS}

Black, B.B., M.W. Collopy, H.F. Percival, A.A. Tiller \& P.G. Bohall. 1984. Effects of low level military training flights on wading bird colonies in Florida. Tech. report no. 7. Fla. Coop. Fish and Wildl. Res. Unit, School For. Res. and Conserv., University of Florida, Gainesville, EEUU.

Brisbin, I.L., C. Collins, G.C. White \& D.A. McCallum. 1987. A new paradigm for the analysis and interpretation of growth data: the shape of things to come. Auk 104: 552-554.
Brody, S. 1964. Bioenergetics and Growth. Hafner, Nueva York, EEUU.

Cubo, J., V. Fouces, M. González-Martín, V. Pedrocchi \& X. Ruiz. 2000. Non-heterochronic developmental changes undelie morphological heterochrony in the evolution of the Ardeidae. J. Evolution Biol. 13: 269-276.

Custer, T.W. \& D.W. Peterson Jr. 1991. Growth rates of Great Egret, Snowy Egret, and Black-crowned NightHeron chicks. Colon Waterbird 14: 46-50.

Custer, T.W., G.W. Pendelton \& R.W. Roach. 1992. Determination of hatching date for eggs of Black-crowned Night-Herons, Snowy Egrets, and Great Egrets. J. Field Ornithol. 63: 145-154.

Davis, W.E. 1993. Black-crowned Night-Heron. In A. Poole \& F. Gill (eds.). The birds of North America No. 74. Acad. Nat. Sci. Philadelphia, PA y Am. Ornithol. Union, Washington D.C., EEUU.

Denis, D. 2001. Dinámica metapoblacional en las colonias de garzas (Aves: Ardeidae) de la ciénaga de Birama, Cuba. J. Carib. Ornithol. 16: 35-44.

Dunn, E.H. 1975. The toming of endothermy in the development of altrician birds. Condor 77: 288-293.

Frederick, P.C. 1997. Tricolored Heron. In A. Poole \& F. Gill (eds.). The birds of North America. No. 306. Acad. Nat. Sci., Philadelphia, PA, and Am. Ornithol. Union, Washington D.C., EEUU.

Freymann, B.P. \& K.L. Schuchmann. 2008. Postnatal growth rates of hummingbirds: review and new records. Wilson J. Ornithol. 120: 884-887.

Gill, F.B. 1998. Ornithology. Freeman \& Co., Nueva York, EEUU.

González-Martín, R., X. Ruiz \& G. Llorente. 1992. Breeding parameters, feeding habits and nestling growth in a recovering population of Purple Heron from the Ebro delta, Spain. Misc. Zool. 16: 147-159.

Hancock, J. \& J. Kushlan. 1984. The herons handbook. Harper \& Row, Nueva York. EEUU.

Hochachka, W. \& J.N.M. Smith. 1991. Determinants and consequences of nestling condition in Song Sparrows. J. Anim. Ecol. 60: 995-1008.

Hudson, J.W., W.R. Dawson \& R.W. Hill. 1974. Growth and development of temperature regulation in nestling Cattle Egrets. Comp. Biochem. Phys. A. 49: 717-741. 
Kushlan, J.A. 1977. Differential growth of body parts in the White Ibis. Auk 94: 164-167.

Lack, D. 1968. Ecological adaptations for breeding in birds. Methuen, Londres, Inglaterra.

Linden, M., L. Gustafsson \& T. Párt. 1992. Selection on fledging mass in the Collared Flycatcher and the Great Tit. Ecology 73: 336-343.

Massemin, S., E. Korpima, V. Poyri \& T. Zorn. 2002. Influence of hatching order on growth rate and resting metabolism of kestrel nestlings. J. Avian Biol. 33: 235-244.

Maxwell, G.R. II \& H.W. Kale II. 1977. Breeding biology of five species of herons in coastal Florida. Auk 94: 689-700.

McVaugh, W.J. 1972. The development of four North American herons. Living Bird 11: 155-173.

Monroes, J., E. Belda \& E. Barba. 2002. Postfledging survival of individual Great Tits: the effect of hatching date and fledging mass. Oikos 99: 481-488.

O'Connor, R.J. 1984. The growth and development of birds. Wiley, Chichester, Reino Unido.

Palmer, R.G. 1962. Handbook of North American birds. Vol. 1: loons through flamingos. Yale University, New Haven, Connecticut, EEUU.

Parsons, K.C. \& T.L. Master. 2000. Snowy Egret. In A. Poole \& F. Gill (eds.). The birds of North America No. 489. Acad. Nat. Sci., Philadelphia, PA, and Am. Ornithol. Union, Washington D.C., EEUU.

Quinney, T.E. 1982. Growth, diet, and mortality of nestling Great Blue Herons. Wilson Bull. 94: 571-577.

Ratkowsky D.A. 1983. Nonlinear regression modeling. Marcel Dekker Inc., Nueva York. EEUU.

Ricklefs, R.E. 1967. A graphical method of fitting equation to growth curves. Ecology 48: 978-983.

Ricklefs, R.E. 1968. Patterns of growth in birds. Ibis. 110: 419-451.

Ricklefs, R.E. 1975. Patterns of growth in birds. III. Growth and development of the Cactus Wren. Condor 77: 34-45.

Ricklefs, R.E. 1979. Adaptation, constraint, and compromise in avian postnatal development. Biol. Rev. 54: 269-290.
Ricklefs, R.E. 1984. Egg dimensions and neonatal mass of shorebirds. Condor 86: 7-11.

Ricklefs, R.E. \& S.C. White. 1975. A method for constructing nestling growth curves from brief visits to seabird colonies. Bird-banding 46: 135-140.

Ricklefs, R.E., J.M. Starck \& M. Konarzewski. 1998. Internal constraint on growth in birds, p. 266-287, In J.M. Starck \& R.E. Ricklefs (eds.). Avian growth and development. Oxford University, Nueva York, EEUU.

Ruiz, X. 1981. Ecología de la Garza Ganadera en el delta del Ebro. Tesis de Doctorado, Universidad de Barcelona, España.

Shanholtzer, G.F. 1972. Range expansion dynamics of the Cattle Egret. Ph.D. Thesis, Georgia University, Athens, EEUU.

Siegfried, W.R. 1972. Breeding success and reproductive output of the Cattle Egret. Ostrich 43: 43-55.

Siegfried, W.R. 1973. Food requirements and growth of Cattle Egrets in South Africa. Living Bird 11: 193-206.

Starck, J.M. \& R.E. Ricklefs. 1998. Patterns of development: The Altricial-Precocial spectrum, p. 3-30. In J.M. Starck \& R.E. Ricklefs (eds.). Avian growth and development: Evolution within the AltricialPrecocial Spectrum. Oxford University, Nueva York, EEUU.

StatSoft, Inc. 2007. STATISTICA (data analysis software system), version 8 . www.statsoft.com.

Telfair, R.C. II. 1983. The Cattle Egret: a Texas focus and world view. Kleberg Stud. Nat. Resour. Tex. Agric. Exp. Stn., Texas A\&M University, College Station, EEUU.

Telfair, R.C. II. 1994. Cattle Egret. In A. Poole \& F. Gill (eds.). The birds of North America No. 113. Acad. Nat. Sci., Philadelphia, PA, and Am. Ornithol. Union, Washington D.C., EEUU.

Thornley, J.H.M. \& I.R. Johnson. 1990. Plant and crop modelling: a mathematical approach to plant and crop physiology. Oxford University, Oxford, EEUU.

Tinbergen, J.M. \& M. Boerlijst. 1990. Nestling weight and survival of individual Great Tits (Parus major). J. Anim. Ecol. 59: 1113-1127. 
Von Bertalanffy, L. 1960. Principles and theory of growth, p. 137-259. In W.W. Nowinski (eds.). Fundamental aspects of normal and abnormal growth. Elsevier, Amsterdam, Holanda.

Werding, G. 1970. Observations on the breeding behavior of Cattle Egrets and Craviers Chevelus, and the growth of the young. Bull. Madagascar 292: 753-768.
Werschkul, D.F. 1979. Nestling mortality and the adaptive significance of early locomotion in the Little Blue Heron. Auk 96: 116-130.

Werschkul, D.F. \& J.A. Jackson. 1979. Sibling competition and avian growth rates. Ibis 121: 97-102.

Winkler, D.W. \& P.E. Allen. 1996. The seasonal decline in Tree Swallow clutch size: physiological constraint or strategic adjustment? Ecology 77: 922-932. 